\title{
Visible spectroscopy of the two largest known trans-Neptunian objects: Ixion and Quaoar
}

\author{
S. Marchi, M. Lazzarin, S. Magrin, and C. Barbieri \\ Dipartimento di Astronomia, Università di Padova, Vicolo dell'Osservatorio 2, 35122 Padova, Italy \\ e-mail: marchi@pd.astro.it;lazzarin@pd.astro.it;s.magrin@pd.astro.it; barbieri@pd.astro.it
}

Received 10 June 2003 / Accepted 28 July 2003

\begin{abstract}
We report visible spectroscopy of the two largest known trans-Neptunian objects (TNOs): Quaoar (=50 000) and Ixion (=28 976) obtained respectively on 4th and 5th May 2003 with the ESO New Technology Telescope (NTT) at La Silla, Chile. The spectrum of Ixion is moderately red with an absorption feature around $0.80 \mu \mathrm{m}$, while Quaoar is a red object with a featureless spectrum. These differences seem to indicate differences in primordial composition or dynamical history, in spite of their present similar orbits.
\end{abstract}

Key words. minor planet - asteroids - trans-Neptunian objects

\section{Introduction}

Ixion and Quaoar are the two largest TNOs known so far. Ixion was discovered in May 2001 (Gnadig et al. 2001), while Quaoar was found in October 2002 (Williams et al. 2002). Their estimated diameters are $1100 \mathrm{~km}$ and $1300 \mathrm{~km}$, respectively. From a dynamical point of view, they belong to the Cubewanos group being their semi-major axes about $39.4 \mathrm{AU}$ and 43.2 AU. Such big objects are very interesting for several reasons.

First of all, they account for the greatest part of the total mass present in the Edgeworth-Kuiper belt (EKb).

Moreover, by considering their dimensions, Pluto could be simply the largest of such objects instead of a bona-fide planet. Finally these bodies could provide new hints about the origin of Triton (Neptune's biggest satellite with a diameter of $2706 \mathrm{~km}$ ), thought to have originated from the EKb.

Previous visible and near-infrared observations of TNOs showed a great variety among them. Their colors range from neutral (or slightly blue) to very red. Some show absorption features, both in visible and NIR spectra, while others are featureless (see for instance Lazzarin et al. 2003; Barucci et al. 1999). The presence of features is of particular interest because from their analysis we can obtain precious information on the composition and evolution of these objects. On the other hand, a red, linear, featureless spectrum would indicate longterm evolution due to solar irradiation, while a neutral color object could suggest a "fresh" surface.

Send offprint requests to: $\mathrm{S}$. Marchi, e-mail: marchi@pd.astro.it

\section{Observations}

The spectra of Quaoar and Ixion have been recorded, respectively, on 4th and 5th May 2003, with the ESO New Technology Telescope (NTT) at La Silla, Chile. The spectrograph was EMMI, used in the low resolution mode with the Grism \#1 and a slit width of $5^{\prime \prime}$. We chose such a wide slit in order to reduce possible tracking problems and/or atmospheric differential diffraction effects, and at any rate the slit was oriented following the parallactic angle. The resolution was about 250 . For each object we recorded two consecutive spectra of 30 min each, with an average seeing for both of $0.7^{\prime \prime}$.

The reduction was carried out with usual reduction techniques, and for details we refer, for instance, to Lazzarin et al. (2003). Particular care has been paid to the correction for atmospheric extinction, recording spectra of different solar analogue starts at different airmasses during both nights. At the end all the solar analogues showed negligible differences. As proof of the accuracy of our method we computed the ratio between the solar analogs' spectra, obtaining a low noise, almost constant curve with a maximun deviation of $5 \%$ around 1 . The ratio shows a $\mathrm{P}-\mathrm{Cygni}$ profile around $0.76 \mu \mathrm{m}$, due to the atmospheric $\mathrm{O}_{2}$, but this artificial feature is restricted to a narrow wavelenght interval and does not affect the behaviour at other wavelenghts. The solar analogues observed are: Landolt 102-1081, Landolt 110-361 and Landolt 107-998.

Moreover, for each object the two consecutive spectra were reduced separately in order to check for the quality of the results. Since they were indistinguishable, we averaged them to 

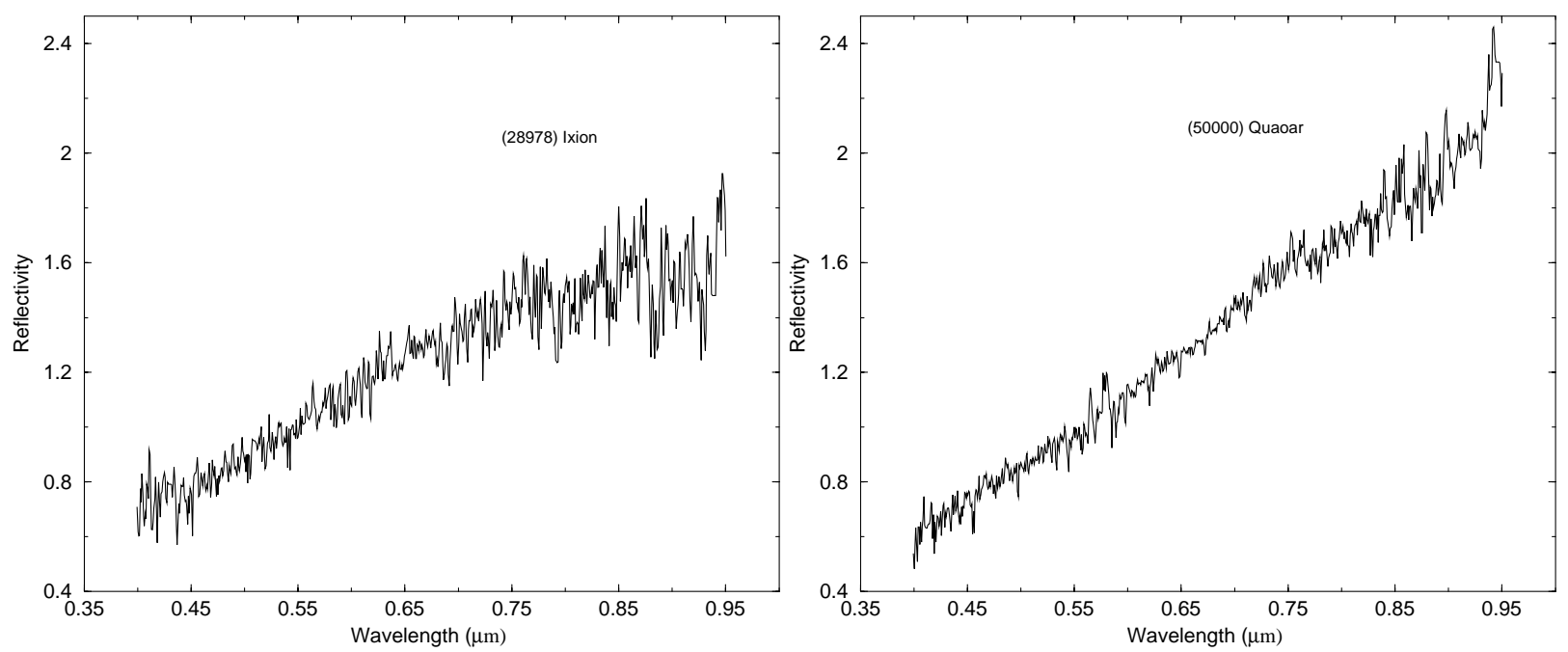

Fig. 1. Reflectivitis of Ixion (left panel) and Quaoar (right panel) normalized at $0.55 \mu \mathrm{m}$.

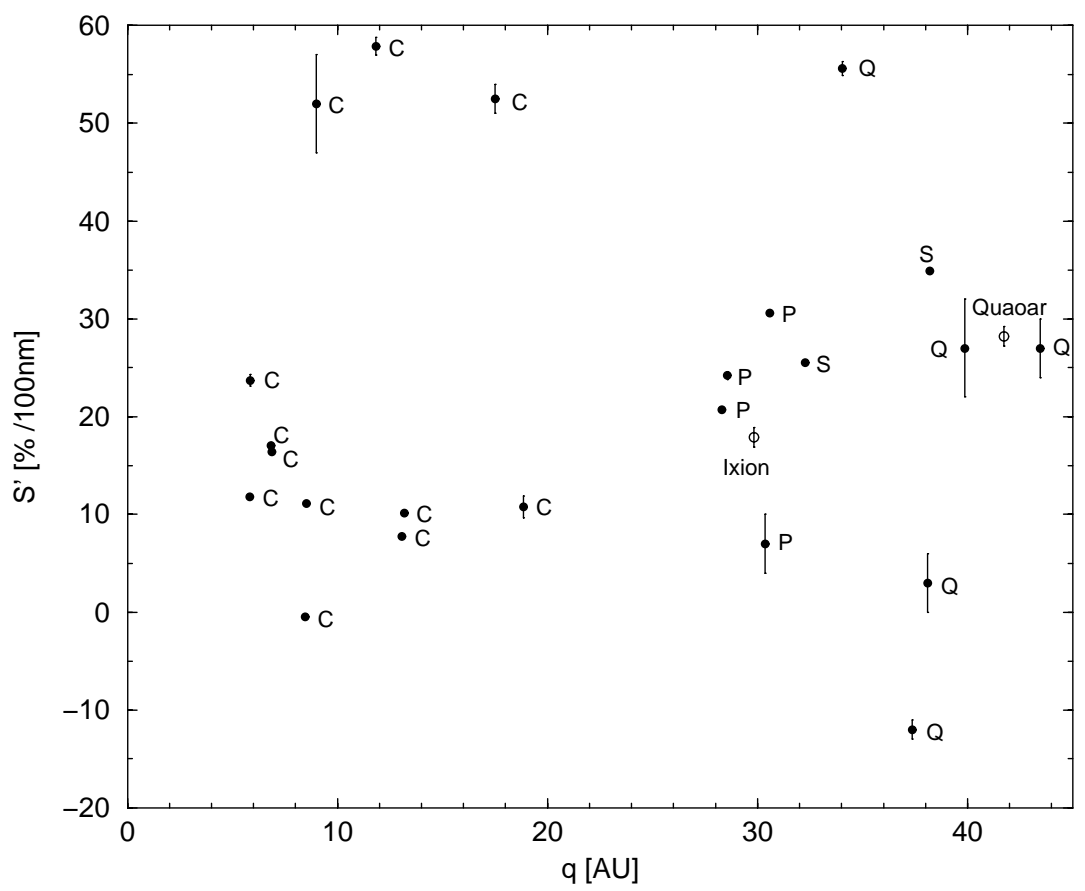

Fig. 2. Reflectivity slope vs. perihelion distance of Quaoar, Ixion and other objects from Lazzarin et al. 2003. "C $\mathrm{C}^{\prime}=\mathrm{Centaurs,} \mathrm{"Q}$ " $=$ Cubewanos, " $\mathrm{P}$ " = Plutinos and " $\mathrm{S}$ " = Scattered.

reduce the noise. The resulting reflectivities are shown in Fig. 1 (both normalized at $0.55 \mu \mathrm{m}$ ).

\section{Discussion}

Ixion. On the basis of the classification presented by Lazzarin et al. (2003) this object shows a moderately red color, and its measured slope in the range $0.4-0.95 \mu \mathrm{m}$ is 17.9 (\% per $100 \mathrm{~nm})$. The spectrum is almost linear shortward of $0.75 \mu \mathrm{m}$ and becomes nearly flat longward of $0.85 \mu \mathrm{m}$ (in agreement with the NIR spectra reported by Licandro et al. 2002). Around $0.80 \mu \mathrm{m}$ it shows an absorption feature about $0.05 \mu \mathrm{m}$ wide. This feature is present if we consider both spectra of the object and the solar analogs. For this reason, although the region around $0.86 \mu \mathrm{m}$ is quite noisy, we are confident of its reality.

Bands in the visible region were found also by Lazzarin et al. (2003) on other TNOs, and attributed preliminarly to water alteration. Also the $0.80 \mu \mathrm{m}$ feature could be attributed to aqueous alteration (see for example Vilas et al. 1994 and Hiroi \& Vilas 1995).

Quaoar. This object shows a red, linear and featureless spectrum with a measured slope of $28.2(\%$ per $100 \mathrm{~nm})$ and it could be included in the red class. The spectra also show a little reduction of slope around $0.75 \mu \mathrm{m}$.

In literature at least two other spectra very similar to that of Quaoar have been observed. These objects are $1997 \mathrm{CQ}_{29}$ 
and $1997 \mathrm{CS}_{29}$, whose radii are about $110 \mathrm{~km}$ and $290 \mathrm{~km}$, respectively (see Boehnhardt et al. 2001). It is quite surprising that bodies with diameter ratios larger than a factor of ten show the same spectral behaviour.

\section{Conclusions}

Although Ixion and Quaoar have similar dimensions, their spectra are quite different. The former can be classified as moderately red object, while Quaoar is a red one. For comparison we report in Fig. 2 the reflectance slope of our two objects as a function of the perihelion distance, as in Fig. 3 of Lazzarin et al. (2003). The measured value for the slope of Ixion is very similar to that of Centaurs instead of that of TNOs.

On the other hand, the spectrum of Quaoar is very close to that of two other Cubewanos, namely $1997 \mathrm{CQ}_{29}$ and $1997 \mathrm{CS}_{29}$. Notice that these three objects lie in the region of the most distant (in terms of perihelion) objects observed spectroscopically (see Fig. 2). Notice that this plot does not provide clear trends yet, maybe for the scarsity of observed objects. However, the Cubewanos range over the full height of the diagram, while the Centaurs seem to be clustered around a slope of 10 (apart from three really red objects).

Moreover, Ixion presents an absorption band around $0.8 \mu \mathrm{m}$ that could be tentatively attributed to aqueous alteration.

Although it is not surprising that such large bodies have red spectra, what is not yet clearly understood is why they show such differences. Most likely they are due to differences in primordial composition or dynamical evolution, in spite of their present similar orbits. For such big bodies the influences of collisional evolution should be not so important.

Finally, we found no relationships between the spectra of the two observed TNOs with Triton (Tryka \& Bosh 1999; Marchi et al. 2003). In particular, the TNOs show no band at $0.43 \mu \mathrm{m}$ and $0.89 \mu \mathrm{m}$ as Triton does, and moreover Triton has a neutral color (measured slope of $2.7 \%$ per $100 \mathrm{~nm}$ ). A possible explanation for that, in case that Triton came really from EKb, could be that Triton was melted during its evolution (see, for instance, Ross \& Schubert 1990). In this case any influence of reddened material would be erased.

\section{References}

Barucci, M. A., Lazzarin, M., \& Tozzi, G. P. 1999, AJ, 117, 1929

Boehnhardt, H., Tozzi, G. P., Birkle, K., et al. 2001, A\&A, 378, 653

Gnadig, A., Helin, E. F., Pravdo, S., et al. 2001, Minor Planet Circulars, Aug. 2001

Lazzarin, M., Barucci, M. A., Boehnardt, H., et al. 2003, AJ, 125, 1554

Licandro, J., Ghinassi, F., \& Testi, L. 2002 A\&A, 388, L9

Marchi, S., Barbieri, C., Lazzarin, M., Owen, T., \& Corsini, E. M. 2003, Icarus, submitted

Ross, M. N., \& Schubert, G. 1990, GeoRL, 17, 1749

Tryka, K. A., \& Bosh, A. S. 1999, Icarus, 142, 571

Vilas, F., Jarvis, K. S., \& Gaffey, M. J. 1994, Icarus, 274

Hiroi, T., \& Vilas, F. 1995, LPS XXVI

Williams, G. V., Helin, E. F., Pravdo, S., et al. 2002, Minor Planet Circulars, Oct. 2002 